\title{
Quantum Emitters in 2D materials
}

\author{
Alexander Solntsev, Igor Aharonovich, and Milos Toth \\ Faculty of Science, University of Technology Sydney, Sydney, New South Wales 2007, Australia.
}

\begin{abstract}
Recently discovered photon sources based on 2D materials are much more practical compared to their earlier counterparts due to high emission rate, robust performance in a range of environmental conditions and ease of photonic integration. It is expected that this platform will make a substantial contribution to a range of quantum optical applications, including quantum communication, computing and sensing.
\end{abstract}

Keywords: photons, quantum optics, 2D materials.

\section{INTRODUCTION}

Photonic integrated circuits that process information encoded in particles of light are poised to revolutionize information processing, communications and sensing. A promising, emerging class of quantum technologies is based on solid state, on demand, single photon emitters (SPEs) coupled to optical resonators and waveguides that serve as building blocks for high density, on-chip quantum photonic circuits ${ }^{1}$. To date, the most investigated solid state SPE systems are epitaxial quantum dots (QDs) that operate primarily at cryogenic temperatures, and color centers. A noteworthy example of the latter is the famous nitrogen vacancy (NV) defect in diamond, which has become a standard for a range of quantum optics experiments and inspired numerous studies of SPEs ${ }^{2}$.

Nevertheless, despite years of research, existing systems are inadequate for real-world applications, and there a significant effort to find high performance emitters hosted by materials that enable integration in solid state, on-chip devices. In the last couple of years, the SPE family expanded upon the discovery of cryogenic quantum emitters in two dimensional (2D) materials $^{3}$, and in 2016 hexagonal boron nitride (h-BN) emerged as a compelling 2D host of SPEs that operate at room temperature ${ }^{4}$. The h-BN system is somewhat analogous to diamond as it has a wide bandgap of approximately $6 \mathrm{eV}$ and can host a broad range of deep trap defects that act as ultra-bright, photo-stable, room temperature SPEs. However, h-BN has the advantage of being atomically thin and therefore offers new possibilities for scientific exploration and device engineering.

\section{APPLICATIONS}

The existing significant level of interest in 2D materials, combined with the ability to perform h-BN experiments at room temperature has led to an outburst of activity over the last year. Rapid progress is being made in photo-physical characterization of SPEs, demonstrations of non-linear excitation and super-resolution imaging of emitters in h-BN, advanced studies of phonon polariton propagation in h-BN and models of the crystallographic structure of potential defects proposed to be responsible for quantum light emission.

The current high rate of progress is likely to not only continue, but to accelerate and produce great advances enabled by the $2 \mathrm{D}$ nature of h-BN. For example, the ability to image individual point defects with atomic resolution by scanning tunneling ${ }^{5}$ and transmission electron microscopy, combined with super-resolution fluorescence imaging ${ }^{6}$ will likely lead to the first direct correlative studies of the atomic structure of quantum emitters in solids. Precise knowledge of defect structure will, in turn, enable progress in first principles simulations of emitter properties and their exploitation in quantum information processing, communication and sensing technologies. 


\section{Potential applications of quantum emitters in 2D materials \\ Quantum computing \\ Quantum communications}

Solid-state quantum emitters are fundamental building blocks of a range of integrated quantum network schemes.

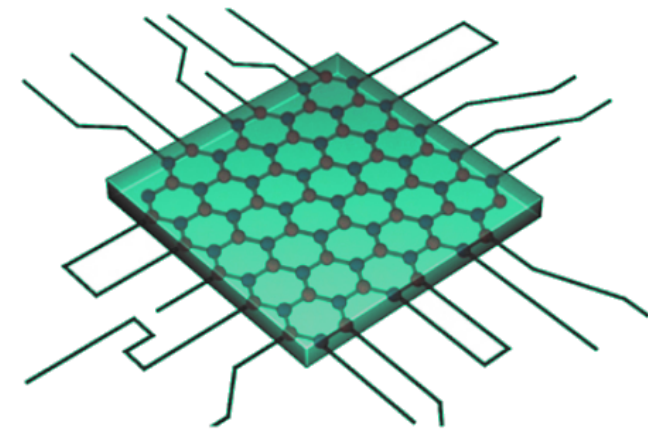

\section{Quantum sensing}

Fluorescent surface defects will enable high-resolution imaging and quantum sensing of biomolecules, analytes, toxins, and adsorbates.

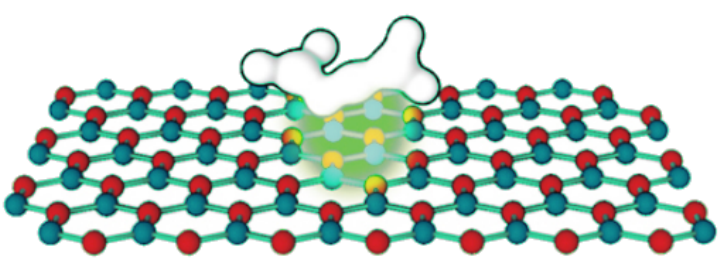

Single-photon sources can encode information, enable quantum cryptography, and secure communications between distant nodes.

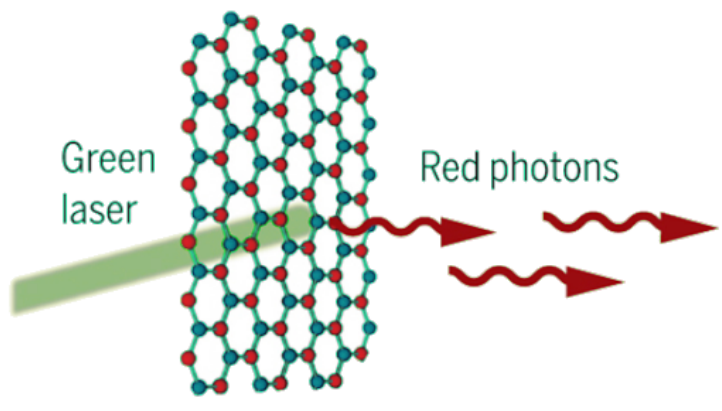

\section{Fundamental science}

Single defects facilitate advanced superresolution imaging modalities and act as sources of quantum light for probing local light-matter interactions at the nanoscale.

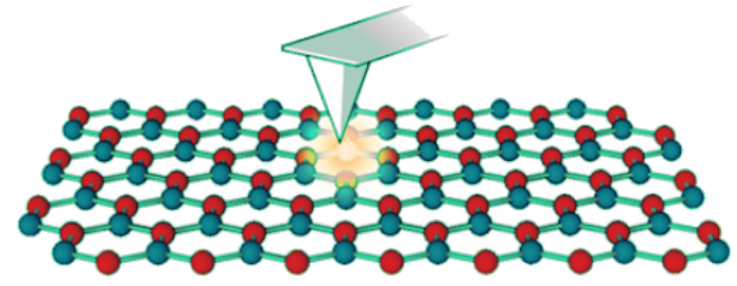

Figure 1. Potential areas of 2D material quantum emitter applications.

\section{FABRICATION AND DEVICES}

In terms of fabrication, electrically triggered emitters have already been demonstrated using 2D TMDCs. The operation of these devices is presently limited to cryogenic temperatures ${ }^{7}$, but h-BN will likely transform this into a room temperature technology. Such proof-of-concept devices represent critical stepping stones towards on-chip quantum circuits, as is coupling of emitters to optical resonators, which has already been achieved with h-BN SPEs and plasmonic structures ${ }^{8}$.

Coupling to dielectric cavities is likely to follow soon given the maturity of pick and place techniques that have been developed for 2D materials ${ }^{9}$, and the fact that h-BN is itself a transparent dielectric. Moreover, the single atom thickness of SPE-containing monolayers will enable unique explorations of nonlinear effects, such as h-BN-based cavity optomechanics ${ }^{10}$ and realization of strong coupling between a color center and a cavity - a long standing goal in the field of quantum optics. 
Nevertheless, despite its vast potential, full exploitation of $2 \mathrm{D}$ h-BN requires dramatic advances in fabrication. Large monolayers of $\mathrm{h}-\mathrm{BN}^{11}$ are sparse, and $\mathrm{h}-\mathrm{BN}$ synthesis is an area where the materials science and quantum photonics communities should come together to develop advanced, new growth protocols designed specifically for quantum applications.

Large quantities of high quality material are pivotal to practical deployment of this unique quantum platform, as are techniques for deterministic engineering of SPEs in h-BN. Defect fabrication can be explored using low energy ion implantation and bottom up synthesis methods based on the achievements made in positioning of atomic defects in 3D crystals such as diamond.

\section{OUTLOOK}

Quantum emitters in h-BN and in other wide bandgap 2D materials are well positioned to become the new playground for rapid exploration of quantum phenomena at the nanoscale. By eliminating most of the surrounding crystal that is responsible for decoherence and background luminescence in 3D materials, 2D systems offer a compelling alternative, with the added benefit of emitter localization in the top-most monolayer, which will ultimately yield improved control over emitter positioning in hybrid coupled systems, sensors and biological media. The integration of 2D quantum hosts with metasurfaces used to control and manipulate light ${ }^{12}$ will lead to conceptually new quantum devices and integrated quantum nanophotonic networks.

Metasurfaces are planar structures that locally modify the polarization, phase and amplitude of light in reflection or transmission, thus enabling lithographically patterned flat optical components with functionalities controlled by design ${ }^{12}$. Transmissive dielectric metasurfaces ${ }^{13}$ are especially important, as most optical systems used in practice operate in transmission, and low losses are especially important for quantum optical applications.

Integration of 2D quantum emitters with metasurfaces is expected to enable detailed characterisation of emission properties through quantum tomography ${ }^{14}$, enhance emission robustness via the use of topological protection ${ }^{15}$ and achieve precise control of single-photon emission properties ${ }^{17}$.

\section{REFERENCES}

[1] Lodahl, P., Mahmoodian, S. \& Stobbe, S., "Interfacing single photons and single quantum dots with photonic nanostructures," Reviews of Modern Physics, 87, 347-400, (2015).

[2] Childress, L. \& Hanson, R., "Diamond NV centers for quantum computing and quantum networks," MRS Bulletin, 38, 134-138, (2013).

[3] "Single photons for all," Nature Nano., 10, 481-481, (2015).

[4] Tran, T. T., Bray, K., Ford, M. J., Toth, M. \& Aharonovich, I., "Quantum emission from hexagonal boron nitride monolayers," Nature Nanotech., 11, 37-41, (2016).

[5] Wong, D. et al., "Characterization and manipulation of individual defects in insulating hexagonal boron nitride using scanning tunnelling microscopy," Nature Nanotech., 10, 949-953, (2015).

[6] Feng, J. et al., "Imaging of optically active defects with nanometer resolution," Nano Lett., 7b04819, (2018).

[7] Palacios-Berraquero, C. et al., "Atomically thin quantum light-emitting diodes," Nat. Commun., 7, 12978, (2016).

[8] Tran, T. T. et al., "Deterministic Coupling of Quantum Emitters in 2D Materials to Plasmonic Nanocavity Arrays," Nano Lett., 17, 2634-2639, (2017).

[9] Novoselov, K. S., Mishchenko, A., Carvalho, A. \& Castro Neto, A. H., "2D materials and van der Waals heterostructures," Science, 353, (2016).

[10] Abdi, M., Hwang, M.-J., Aghtar, M. \& Plenio, M. B., "Spin-mechanics with color centers in hexagonal boron nitride membranes," Phys. Rev. Lett., 119, 233602, (2017). 
[11]Chang, R.-J. et al., "Growth of Large Single-Crystalline Monolayer Hexagonal Boron Nitride by OxideAssisted Chemical Vapor Deposition," Chemistry of Materials, 29, 6252-6260, (2017).

[12]Hou-Tong, C., Antoinette, J. T. \& Nanfang, Y., "A review of metasurfaces: physics and applications," Reports on Progress in Physics, 79, 076401, (2016).

[13] Kuznetsov, A. et al., "Optically resonant dielectric nanostructures," Science, 354, aag2472, (2016).

[14]Wang K. et al., "Quantum Imaging with Dielectric Metasurfaces for Multi-Photon Polarization Tomography," Conference on Lasers and Electro-Optics, JW4G.5, (2017).

[15] Barik S. et al., "A topolgical Quantum Optics Interface," Science, 359, 666-668, (2018).

[16] Kruk S. et al., "Broadband highly efficient dielectric metadevices for polarization control," APL Photonics, 1, 030801, (2016). 